\title{
UNA APROXIMACIÓN ONTTOLÓGICA A LA REALIDAD FÍSICA
}

\author{
ADOLF HÜBNER \\ INSTTTUT FÜR WITTGENSTEINFORSCHUNG, \\ Analytische Philosophie und Wissenschaftstheorie \\ Austrian LudWig Wittgenstein SOCIETY
}

To propongo que se considere si as pasible, $y$ de ser asi, qué es posible inferir de la estructura del lenguaje sobre la estructura del mundo. -Personalmente, yo creo que, on parte mediante el estudio de la sintaxis, podemos alcanzar un conocimiento considerable sobre la estructura del musudo.

Bertrand Russell ${ }^{1}$

\section{PRIMERA PARTE \\ SOBRE EL MÉTODO FILOSÓFICO: LA CONTROVERSIA RUSSELL-WITTGENSTEIN}

\section{Introducción}

El conflicto Russell-Wittgenstein sobre la "posibilidad de la filosofia" tiene como antecedente la discusión respecto a si es o no factible hablar de la existencia de un método filosófico. Es obvio que la existencia de un método filosófico constituye la primera condición para que la filosofía sea posible como actividad intelectual razonable, es decir, como una actividad que puede llevar a un incremento del conocimiento de la estructura del mundo, que resulta tanto más prometedora cuanto más difiere del razonamiento científico (convencional). La trascendencia de la discusión entre Russell y Wittgenstein en el contexto de la historia de las ideas radica, sin lugar a dudas, en el hecho de que el tema referente a la posibilidad de la filosofía fue colocado en su lugar, es decir, "fue claramente articulado". Para decirlo en pocas palabras: la filosofía, en tanto actividad racional por derecho propio, es concebible siempre y cuando exista una posibilidad, no vaga, de inferir de la entidad "estructura del lenguaje" - abstracta, por empírica que sea - la entidad, también abstracta, "estructura del mundo" (punto de vista russelliano).

1 “An Inquiry into Meaning and Truth”, Londres, 1987, p. 341. 
Mientras que en la proposición 5.5563 del TLP Wittgenstein dice estar de acuerdo con el concepto russelliano en cuanto a la existencia de una estructura lógica del lenguaje, en el párrafo 97 de las Investigaciones filosóficas declara que la idea de la existencia de un "orden de posibilidades que debe ser común tanto al mundo como al pensamiento" es una quimera.

Esto es lo que llevó a Russell a decir: "El último Wittgenstein parece haberse cansado del pénsamiento serio y haber inventado una doctrina que haría innecesaria tal actividad".

En el presente artículo, el autor intenta demostrar la exactitud del concepto russelliano, presentando un "éxito ontológico", bajo la forma de un resultado sintético ("no científico"), que no contradice el conocimiento físico actual y que, consecuentemente, refuta el enfoque wittgensteiniano por ser lógicamente insostenible.

Inserción: En tanto que los postulados de Russell - siendo positivos- contienen la posibilidad de ser, por principio, lógica y empíricamente demostrables, las afirmariones escéptico-agnósticas de Wittgenstein -como todo postulado negativo - no dieron nunca cabida a la posibilidad de una comprobación lógica. ( $\mathrm{Y}$ esto se debe, simplemente, al hecho de que no existen situaciones "lógicamente negativas" y, en consecuencia, que lo "lógicamente negativo", es decir, los argumentos escéptico-agnósticos, no existen.) Me inclino a pensar que si tanta gente considera "sensacional" el trabajo de Wittgenstein, esto se debe a que él presenta como si fuera un hecho el "descubrimiento" de que las tendencias naturales del juicio lógico del hombre -incluyendo las de Russell- no son sino fantasías intelectualmente engañosas. Yo, junto con Russell, "voy a suponer la existencia de un lenguaje lógico".

\section{Wittgenstein y la "pregunta incomparable"}

El primero de junio de 1915, Wittgenstein escribe en sus Cuadernos: "El gran problema alrededor del cual se centra todo lo que escribo es: "¿Hay acaso un orden a priori en el mundo, y, si así es, en qué consiste?"'.

Con esta temprana afirmación, Wittgenstein destaca la singularidad del problema, cuya solución constituye el principal objetivo de la filosofía. De hecho, la filosofía, en tanto actividad racional, no puede tener otro propósito que el de responder a esta pregunta incomparable.

Muchos tienen la intuición de que el mundo es una unidad sometida a un orden a priori, uniforme y único. Intentar comprender un supuesto orden $a$ priori del mundo significa, antes que nada, tratar de encontrar un cierto tipo de legalidad que pudiera subyacer al mundo fenoménico y determinarlo.

Suponiendo que el carácter del orden a priori del mundo ya ha sido identificado, surge entonces la pregunta de cómo puede este orden determinar y guiar el mundo de los fenómenos. Así pues, queda claro que la respuesta que 
la filosofía intenta dar al enigma del mundo es muy diferente a la que buscan los científicos mediante la aplicación de métodos científicos.

Podemos decir que la filosofía tiene propósitos más ambiciosos que la ciencia. No desea una simple "fórmula del mundo" que permanezca dentro del aspecto del mundo como cosa, sino que intenta hallar y acceder a su estructura metafísica.

$\mathrm{Si}$-y sólo si- se puede demostrar que la manifestación y dependencia del mundo fenoménico se dan dentro de un orden a priori central, singularmente dado y racionalmente comprensible, que es tanto uniforme como no axiomático, se accede realmente a los problemas de la vida humana.

Hoy en día, cuando los científicos buscan una "fórmula del mundo", es decir, una fórmula que explique "de una vez por todas" la totalidad de la realidad física, de hecho no desean dar una respuesta a nuestros problemas de la vida, ni es tampoco posible que una respuesta concebida como fórmula del mundo toque dichos problemas. A esto se refiere Wittgenstein cuando escribe:

Sentimos que aun cuando todas las preguntas cientificas posibles fueran respondidas, no se habrian siquiera tocado los problemas de la vida. Por supuesto que cntonces ya no quedarian más preguntas y esto on si seria la respuesta. (TLP, 6.52)

Wittgenstein no sólo abandonó muy pronto su búsqueda de un orden a priori de las cosas del mundo, sino que la negó. El 3 de septiembre de 1914, escribe:

Bueno, si todo aquello que requiere ser demostrado lo es a través de la existencia de las ORACIONES Sujeto-Predicado, etc., entonces la tarea de la filosofia es diferente de la que había supuesto originalmente. Sin embargo, si éste no es el caso, 'lo que falta' tendría que ser mostrado por 'algún tipo de experiencia', y juzgo que esto es imposible... No existe ningún orden a priori de las cosas... todo lo que podemos describir podría ser diferente de lo que es. (TLP, 5.634)

Sin embargo, la proposición 5.634 del TLP acaba de tajo con aquello que, expresado en el punto 5.563 , constituía el foco de su atención:

De hecho, todas las proposiciones de nuestro lenguaje cotidiano, tal como aparecen, se encuentran en un orden logico perfecto. No es que eso tan simple que tenemos que formular aquí se asemeje a la verdad, sino que es la verdad misma, en su totalidad. (Nuestros problemas no son abstractos sino quizá la cosa más concreta que existe.)

"El formular eso tan simple, con el fin de resolver nuestros problemas" se vuelve, además, imposible con la proposición 4.0312, que dice: 
La posibilidad de la proposición descansa en el principio de que los objeros tienen signos que son sus representantes. Mi idea fundamental es que las 'constantes lógicas' no son representantes; que no puede haber representantes de la lógica de los hechos.

No es sino hasta el párrafo 97 de las Investigaciones filosofficas cuando Wittgenstein hace total su negación de la filosofia, al negar la existencia del "orden de posibilidades que debe ser común tanto al mundo como al pensamiento". Pero ahora, entonces, se vuelve totalmente oscuro el significado del concepto de "Lógica de los hechos"(TLP, 4.0312), si éste no significa el orden a priori de los hechos.

\section{Digresión: de la forma en que Wittgenstein acaba con la "conceptualidad verbal"}

En una gran cantidad de casos - aunque no en todos-en que usamos la palabra 'significado', ésta puede ser definida de la siguiente manera: el significado de una palabra es el de su uso en el lenguaje. ( $I F, 43$; subrayado mío)

Conocer el significado de una palabra significa urilizarla en la misma forma en que lo hacen los demás. . . (usarla) 'de manera correcta'... eso no significa absolutamente nada. (Vorlesungen uber die Grundlagen der Mathematik, Cambridge, 1939; subrayado mío)

Lo que nosotros hacemos es traer la palabra del plano metafísico a su uso cotidiano. $(I F, 166)$

Si unimos el primer postulado con el segundo, resulta claro que Wittgenstein considera que la comunidad de usuarios del lenguaje podría reconocer el uso incorrecto de una palabra en cierto contexto, con toda seguridad, en todo caso imaginable y bajo cualquier circunstancia.

Lo que Wittgenstein deja de lado es, primero que nada, la posibilidad de que puedan existir razones (criterios) que impidan la utilización de una palabra en un contexto dado, si el significado de dicha palabra es cuidadosamente tomado en consideración en su relación con ese contexto; y más adelante niega que la falta de reflexión sea una cualidad usual de la gente. Dentro de la difundida "propensión a la superficialidad", puede en efecto existir una tendencia común en las personas a hacer un uso erróneo de las palabras. Sin embargo, en los casos ontológicamente importantes, el uso equivocado de un solo predicado puede ser desastroso.

El lenguaje humano natural es un fenómeno único e incomparable en el mundo, y lo mismo se aplica a su desarrollo. Desde el punto de vista de una Weltanschauung adecuada, no existe nada más importante que el uso de la palabra "desarrollo" en relación con el desarrollo del lenguaje. 
La palabra "desarrollo" tiene un significado activo cuando decimos: "Existe un posible agente (una persona) que desarrolla algo a partir de algo disponible, con base en ciertas ideas". Cuando se habla de la existencia de tales agentes, siendo éstos criaturas, es imposible decir que ellos se han desarrollado a sí mismos, lo cual incluye, por supuesto, todas sus cualidades. Los agentes humanos son producto del mundo que, junto con sus potencialidades, de alguna manera surgieron. Es imposible decir que, en épocas remotas, el hombre tuvo la idea de desarrollar el lenguaje y que, además, tenía los conocimientos necesarios (que suponen ya la existencia de pensamientos ordenados en forma semejante al lenguaje) para desarrollarlo.

Dado que Wittgenstein nunca tomó en consideración la evolución del lenguaje, sino sólo el aprendizaje del ya existente, no le era posible observar que a su idea de que el lenguaje se origina y existe por consenso se le escapa el hecho de que la producción activa del lenguaje conceptual, por el hombre, en el curso de su evolución, es imposible. El "consenso" no es un principio de creación sino solamente un principio de coordinación para todo lo que en lenguaje puede ser arbitrario, principalmente el vocabulario. Lo que, cuando mucho, se puede decir, es que el lenguaje, junto con sus regularidaes sintácticas, se ha desarrollado sobre el sustrato "hombre", a partir de las facultades comunes a todos los hombres, las cuales les fueron concedidas en total correspondencia con las cualidades abstractas del mundo.

Cuando Wittgenstein identifica el significado de una palabra con su uso en el lenguaje, y dice que lo que él hace es traer las palabras del plano metafísico a su uso cotidiano, lo que de hecho hace es erradicar la conceptualidad textual, la cual determina, en sentido lógico, el uso correcto o incorrecto de un predicado ligado a un determinado sustantivo.

\section{La filosofia como actividad racional}

Declarar que la existencia de un método filosófico es lógicamente imposible significa dejar a la filosofía sin esperanza alguna de tener éxito como actividad racional. De ahí que Russell acusara a Wittgenstein de ser un traidor a la filosofía. A los ojos de Russell, Wittgenstein hacía trucos para abrir todas las puertas al escepticismo ilimitado, hoy conocido con el nombre de "posmodernismo".

La filosofía, como actividad racional diferente de la ciencia, requiere de un método que sea suyo propio. Dado que la filosofía intenta responder a nuestros "problemas de la vida" de manera distinta a la forma en que lo hacen las ciencias naturales, la pregunta que surge es: ¿qué tipo de procedimiento es el aplicable para que la filosofía sea exitosa de forma diferente a la ciencia y la trascienda lo suficiente para que pueda abordar los problemas de la vida humana? 
Russell no tuvo ninguna dificultad en identificar el punto de partida del método filosófico. Para él era claro que el estatuto diferenciado del lenguaje humano, como entidad empírica que pertenece a la realidad, debía posibilitar el llegar a un conocimiento seguro de la estructura metafísica del mundo, a través de los rasgos lógicos idénticos que se hallan detrás tanto del mundo como del lenguaje.

Esto es lo que Russell llama su "teoría de la verdad como correspondencia". Escribe:

Existen casos cuya descripción verbal requiere de oraciones del tipo 'a es similar a $b$. Pero este hecho lingǘrtico parece implicar un hecho acerca de los casos descritos, es decir, el tipo de hecho que se asevera cuando digo ' $a$ es similar a $b$ '. Cuando digo: 'existe la similitud', es este hecho acerca del mundo, y no algo acerca del lenguaje, lo que deseo aseverar. Ĺa palabra 'amarillo' es necesaria puesto que existen cosas amarillas; la palabra 'similitud' es necesaria porque existen pares de cosas similares. Y la similitud de dos cosas es un hecho no-lingǘstico tan real como el color amarillo de una cosa. Hemos llegado en este capítulo -prosigue Russell- a un resultado que, en cierto sentido, ha sido el objeto de toda nuestra discusión. El resulmdo que tengo en mente es el siguiente: que el agnosticismo metafísico es incompatible con la afirmación de las proposiciones lingüísticas. Algunos filósofos modernos sostenían que sabemos mucho sobre el lenguaje pero nada acerca del resto. Este punto de vista olvida que el lenguaje es un fenómeno empírico como cualquier otro, y que aquel que es un agnóstico metafísico debe negar que sabe cuándo usa una palabra. Personalmente, yo creo que, en parte a través del estudio de la sintaxis, podemos alcanzar un conocimiento considerable sobre la estructura del mundo. ("An Inquiry into Meaning and Truth", Londres, 1980, p. 347)

\section{El callejón sin salida de Russell}

Si bien estamos de acuerdo con la "teoría de la verdad como correspondencia", considerando que es el punto de partida correcto - frente al cual simplemente no se puede pensar en otra alternativa-, debemos decir que, en nuestra opinión, él nunca propuso una "teoría". Quien propone una teoría y después no puede hacer otra cosa más que aconsejar el volverse "hacia a el estudio de la sintaxis", no ha propuesto una teoría sino sólo una idea vaga.

Una teoría que no ofrece guías para proceder de forma que exista la posibilidad de lograr resultados exitosos en uno u otro sentido, carece de la esencia misma de lo que puede llamarse una teoría. Esto quiere decir que la oración de Russell: "La similitud existe como hecho lingüístico y como hecho no linguístico" no muestra poder sintético alguno, lo cual implica que no puede hacerse de ella la base de un método filosófico.

La "teoría de la verdad como correspondencia" de Russell requiere ser completada con guías que muestren, en concreto, cómo y con qué base se pueden 
efectuar inferencias, a partir de la estructura del lenguaje, sobre la estructura del mundo.

Observación: Si consideramos la controversia Russell-Wittgenstein desde la perspectiva de su "importancia contributiva", podemos, en un muy particular sentido, afirmar que la clara exposición de Wittgenstein del enfoque -para nosotros-erróneo, tiene mucho más valor que la exposición que hace Russell del enfoque correcto. Una negación clara pone a la vista las alternativas, en tanto que una afirmación vaga sólo tiene un vago valor epistemológico.

\section{SEGUNDA PARTE \\ LA ONTOLOGLA COMO CIENCIA}

\section{La formación de la "teoria de la verdad como correspondencia" de Russell}

Considerando que el lenguaje humano natural posee un estatuto ontológico, es posible que exista algún medio para aumentar nuestro conocimiento sobre la estructura de nuestro mundo, de tal suerte que, siendo éste científico, sea a la vez diferente del razonamiento científico convencional.

- Sostenemos un estatuto ontológico para la estructura del lenguaje natural humano, toda vez que éste es un producto del mundo - como el hombre mismo-, que surgió de alguna manera.

- El estatuto ontológico descendiente del lenguaje humano natural se confirma por su propia estructura lógica, ya que es imposible decir: "el hombre desarrolló el lenguaje".

- El uso de la palabra "desarrollo" supone que cualquiera que desarrolle algo en un sentido creativo debe disponer de una comprensión conceptual consciente de cómo (de acuerdo con qué reglas) aquello que se desarrolla funcionará en el futuro, es decir, después de su realización. Es claro que no se puede afirmar que los primitivos ancestros de la raza humana tuvieron, sobre la entidad abstracta "lenguaje humano", un conocimiento explícito de las reglas sintácticas que se habrian de usar en el futuro.

- Una estructura abstracta de la que, necesariamente, se debe pensar que fue "originada" por una estructura madre, sin intervención alguna, puede darse solamente a partir de una correspondencia estructural no parcial. Afirmamos: la estructura del mundo y la estructura del lenguaje forman un sistema evolutivo auto-referencial (cibernético) dentro del aspecto abstracto del mundo.

- Se puede perfectamente hablar de una evolución del aspecto abstracto del mundo hacia la autoconciencia.

- Un sistema auto-referencial permite inferir la estructura antecedente a partir de la estructura descendiente. 
- Nosotros vamos a dar forma a la "teoría de la verdad como correspondencia" de Russell, afirmando: aquello que es impensable -y, por ende, inexpresable por el lenguaje - es imposible como cualidad fisica de la realidad.

- Los signos y las relaciones de los signos, en el lenguaje humano natural, representan los objetos del mundo y sus relaciones posibles, por medio de lo "pensable"y lo "impensable".

- Buscamos sobrepasar lo sintáctico, es decir, buscamos aquellas condiciones estructurales lógico-sintácticas dentro del lenguaje que pueden ser utilizadas para formular una cadena de postulados racionales sobre la estructura del mundo.

- Una condición aún más importante, que niega la posibilidad de que exista una multitud de lógicas con respecto al tema de la "realidad física", está dada por la inexpresabilidad de la oración: "Se puede hablar de una diversidad de lógicas que determinan el modo en que funciona un todo estructuralmente homogéneo".

La homogeneidad estructural requiere de una uniformidad lógica.

\section{Alfa: una pieza aún no denotada de la metafisica}

- Un mundo es un mundo justamente por el hecho de que todos sus estados no obedecen sino a una única lógica no-axiomática, singularmente dada, es decir, a un único orden a priori. ${ }^{2}$

- Un mundo que es racional debe ser construido a partir de un plan.

- Si es así, debe existir un plan natural, es decir, un plan que está en las manos de la naturaleza y de acuerdo con el cual el mundo se despliega fenoménicamente.

- El mundo muestra números no dimensionales, como la constante universal $\alpha$ y la razón de la masa del protón a la masa elemental, etc. Con ello se demuestra que, más allá de toda duda razonable, el mundo posee un aspecto lógico-numérico, lo cual quiere decir que el mundo es una materia de números naturales y sus vínculos naturales. (El mundo es un caso no algebraico.)

- A lo largo de la evolución de la física, formando parte de una descripción correcta de la realidad física, la constante estructural $\alpha$ ha aparecido como número no dimensional. En tanto entidad abstracta no dimensional, que adopta la forma de un número, ella constituye una evidencia abstracta de la existencia de un aspecto lógico-numérico del mundo que, como un a priori, se

2 Hübner, A., "World's Order A Priori and its Logico-Mathematical Foundations", Kirchberg am Wechsel, 1988 (sin publicar); “Über den Zusammenhang des physischen und metaphysischen Aspektes der Wirklichkeit”, Kirchberg am Wechsel, 1988 (sin publicar); “A Uniform Rational Theory of Physics”, Kirchberg am Wechsel, 1988 (sin publicar). 
halla detrás de las regularidades de la realidad existente, que aparecen bajo la forma de leyes de la naturaleza.

- Podrá mostrarse la característica de $\alpha$ de ser un vínculo singularmente dado entre los aspectos físico y metafísico del mundo si somos capaces de abordarla desde el lado del aspecto metafisico de la realidad.

- Nuestro objetivo es establecer un contexto matemático conceptualmente bien fundamentado -en un proceso "no científico" de mera operación del pensamiento-, esperando que $\alpha$ haga acto de presencia al aplicar a este contexto cierta proposición básica de la fisica "que reclama identidad sustancial".

- Nuestra esperanza es que la manifestación de $\alpha$ en la metafísica tenga un carácter reciproco con respecto al lenguaje objeto, en comparación con lo que sucede al aparecer en la fisica: en tanto que esto último se asociaba con la desaparición del lenguaje objeto - desaparecían los signos dimensionales-, esperamos que su manifestación en la metafísica vaya acompañada de la aparición del lenguaje objeto, bajo la forma de un signo dimensional bien definido.

- Si ello sucediera, es decir, si bajo las condiciones de "magnitud semejante a esto" de nuestras unidades de medición, apareciera la dimensionalidad, se demostraría que nuestro sistema de medición no sólo era aplicable a la descripción de la realidad física, sino que es correcto en sentido logico.

- Si ahora consideramos que podríamos haber usado un sistema de medición con la misma forma lógica del nuestro, pero con unidades de medición con "magnitud semejante a eso" muy diferente, resulta muy probable que la "semejanza a uno" (Einsmäßigkeit) de las unidades de medición sea la uinica condición lógica para poder discernir la naturaleza del a priori metafísico de la realidad existente.

\section{Esquema del desarrollo argumentativo que se presenta en este trabajo}

El curso de nuestra argumentación puede resumirse de la siguiente manera: Postulado No. 1: El sistema de los logaritmos naturales representa el orden natural único de los números naturales.

- El sistema de los logaritmos naturales es único. (Todos los matemáticos, en cualquier tiempo y lugar del cosmos, deben necesariamente llegar a $e$, y, si utilizan el sistema decimal de números, llegarán a nuestra $e$ decimal.) Postulado No. 2: La manifestación del sistema de logaritmos naturales (S) proporciona la base numérica (las guías) para la manifestación del mundo fenoménico $(\mathrm{R})$.

- e es la letra-código decimal de ese plan;

- e sólo tiene dos cualidades:

a) es una secuencia de números con forma única (irrepetible), que 
b) se expresa en el sistema decimal de números.

- Dado que el número e representa la base inalterable de su poder para generar el sistema de los logaritmos naturales y -al igual que supusimos sobre la manifestación del mundo fenoménico- sólo a su cualidad de "ser decimal" se puede atribuir la capacidad de establecer lineamientos numéricos para el mundo de los fenómenos;

- Suponiendo que todo aquello que se manifiesta y que puede ser comprendido de manera racional debe, para hacerlo, dar el primer y más simple paso, esto nos lleva a afirmar que $e^{0,1}$, siendo el primer paso decimal de $e$ superior a uno, es necesariamente el primer paso a dar para hacer manifiesta la realidad fenoménica. La pregunta es: ¿cómo?

- Partiendo de que los hechos numéricos no dimensionales que presenta una descripción correcta del mundo (la física) son proporciones, concluimos que $e^{0,1}=1,1051709$ probablemente demuestre su poder para marcar guías numéricas sobre la realización del mundo fenoménico, si podemos representarla en la forma de una proporción, es decir, en la forma de un contexto geométrico que se base en ella.

- Tenemos la posibilidad de lograr un "éxito ontológico" si el resultado de dicho contexto geométrico basado en $e^{0,1}$ es tal que se le pueda aplicar una de las proposiciones básicas de la física que "reclaman identidad sustancial". Es justamente en ese caso cuando podría esperarse que se manifestara una "incógnita" calculable.

- Lo que necesitamos es un contexto que presente tanto poder sintético como explicativo, con respecto a ciertos hechos científicos del mundo fenoménico. Nuestro objetivo es llegar a la síntesis de una proposición básica de la física y nuestro contexto geométrico.

\section{Concretización de una "representación" ontológica}

Si bien el poder de $e$ para formular leyes físicas se ha hecho muchas veces evidente en la ciencia física, es necesario hacer ciertas consideraciones conceptuales si queremos utilizarla con un poder decimal.

Dado que lo que buscamos es que aparezca el número decimal de $\alpha$, parece razonable tomar en consideración la "decimalidad" de nuestro sistema de números, en el más estricto sentido, es decir, en forma de los poderes decimales hacia $e-y$ de hecho no vemos una alternativa a este procedimiento. ( $\mathrm{Si}$ en verdad existe alguna manera para llegar, a partir del sistema de logaritmos naturales, a la constante estructural decimal de la realidad, son tan sólo los poderes decimales de $e$ los que nos mantienen estructural $y$ formalmente dentro de la decimalidad.)

Representamos $e^{0,1}$ como la razón de dos ángulos en el círculo unitario, y el resultado es el siguiente: 


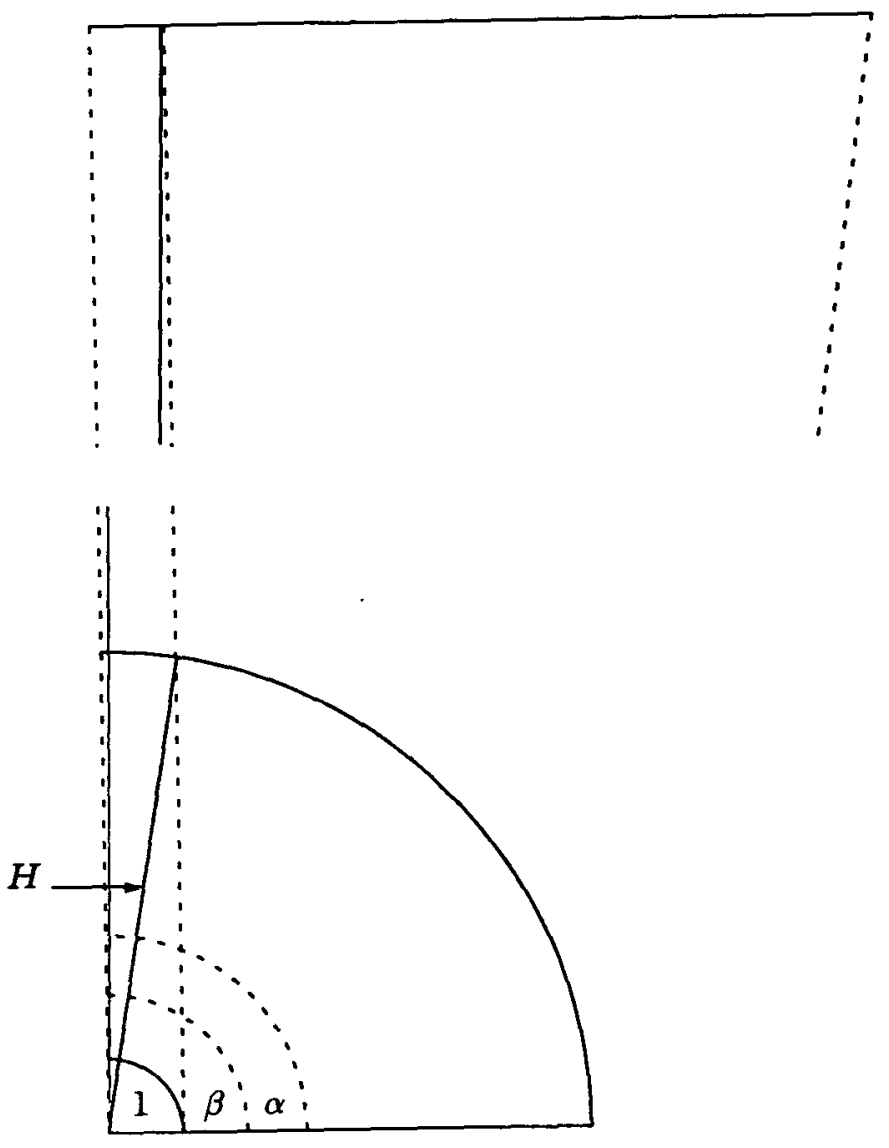

Figura 1

$$
\begin{aligned}
e^{0,1} & =1,1051709 \\
\alpha / \beta & =89,048978 / 80,574852 \\
& =e^{0,1} \\
& =1,1051709 .
\end{aligned}
$$

Razón de catetos opuestos: 60,241:6,0241 = 10:1 (exactamente). Longitud de la hipotenusa del triángulo menor: $\mathrm{H}=6,1065359$ (Matemáticamente hablando, hemos aplicado la teoría de las funciones reales.)

- Dado que los triángulos rectángulos pueden usarse para representar el fenómeno relativista de la dilatación del tiempo, procedemos de la siguiente 
manera: dividimos $\mathrm{H}$ entre $c$ (velocidad de la luz) y llegamos al valor recíproco de una velocidad $\left(1 / v_{0}\right)$, el cual invertimos con la ayuda del cateto " 1 " de nuestros dos triángulos: $v_{0}=4,9093711 \times 10^{9} \mathrm{~cm} / \mathrm{seg}$.

- El estado más simple de energía que podemos establecer mediante la ayuda de $v_{0}$ es $m_{e 0} v_{0}{ }^{2}$, es decir, una cantidad de energía en donde $m_{e 0}$ representa la masa elemental.

- Dado que todo valor de energía expresado por $m v^{2}$ puede ser igualado a un valor de energía $\mathrm{eV}$ (en donde $e$ es la carga eléctrica elemental y $V$ una tensión eléctrica), nuestro contexto geométrico-numérico muestra una facultad sintética, por la ecuación:

$$
m_{e 0} v_{0}^{2}=e V .
$$

- $V$ aparece como una "incógnita" calculable $(x)$.

De esto resulta que la magnitud de $V$ es:

$$
\begin{aligned}
V & =1,37035953 \times 10^{12} \sqrt{\mathrm{gcm}} \sqrt{\mathrm{cm}} / \mathrm{seg}^{2} \text { (voltios corregidos) } \\
& =(1 / \alpha) \times\left(10^{10} \sqrt{\mathrm{g}} \mathrm{cm} \sqrt{\mathrm{cm}} / \mathrm{seg}^{2}\right)
\end{aligned}
$$

Nota: el valor de $\mathrm{l} / \alpha$ que ha aparecido en nuestro contexto concuerda perfectamente con el valor estimado experimental actual, que es de $1,37035963 \pm$ $0,000015 .^{3}$

- La energía esencial (en reposo) del electrón, $m_{c 0} c^{2}$, expresada como energía eléctrica, puede escribirse de la siguiente manera:

$$
\begin{aligned}
m_{c 0} c^{2}= & e V e=e \times\left(H^{2} / \alpha\right) \times 10^{10} \\
= & 1,6021917 \times 10^{-20} \sqrt{\mathrm{g}} \sqrt{\mathrm{cm}} \times 5,11042 \\
& \times 10^{13} \sqrt{\mathrm{gcm}} \sqrt{\mathrm{cm}} / \mathrm{seg}^{2} \\
= & 8,1872647 \times 10^{-7} \mathrm{erg} .
\end{aligned}
$$

Podemos decir que hemos logrado nuestro propósito de propiciar la aparición de $\alpha$ en nuestra estructura ontológica, al utilizar una de las cuatro proposiciones de la física que reclaman identidad, respondiendo así a nuestras expectativas conceptuales.

\section{Interpretación preliminar del resultado obtenido}

Al) Al parecer, se confirma nuestro postulado de que el mundo, siendo una totalidad lógica, no admite sino un solo orden lógico.

3 Particle Data Group, Physics Letters, 107B, 1986, pp. 1-350. 
A2) El orden a priori al que las cosas del mundo se someten es el orden natural de los números.

A3) Se puede hablar de una logica de los hechos, dado que se ha podido demostrar que los hechos de la realidad dependen del orden numérico natural representado por el sistema de los logaritmos naturales.

A4) Se pudo demostrar que es posible trasladar el orden no espacial y atemporal de los números a la lógica de la geometría, a través de una función diferenciada, que nos permitió considerar la máxima decimalidad posible de nuestro sistema numérico.

A5) Habiendo sido necesario hacer uso de uno de los principales rasgos de la Teoría de la Relatividad Especial, se demuestra que la relatividad es una condición logica y necesaria de la realidad física existente. Más aún, la interpretación relativista del triángulo rectángulo de nuestra figura muestra hasta qué punto la "dinámica" se relaciona y puede surgir de la "estática". (Las longitudes de los lados de los triángulos rectángulos se pueden interpretar como resultado del producto dinámico "velocidad $\times$ tiempo".)

A6) La realización de una dimensionalidad puramente decimal, que aparece bajo la forma de la dimensión de una tensión eléctrica como factor de $\alpha$, abre la posibilidad - como será mostrado más adelante- de introducir, sin mayores dificultades, ese fundamental fenómeno físico dentro del aparato formal de la mecánica cuántica.

B) Más aún, las características de nuestros resultados sugieren que el uso de unidades de medición arbitrarias para la longitud, la masa y el tiempo, no pueden impedir la manifestación de la estructura lógico-numérica de la realidad física, bastando con que se dé la "semejanza a uno" de las unidades de medición.

Cl) Finalmente, los resultados de nuestra representación ontológica han dejado claro que se puede hablar de una evolución sintáctica abstracta del mundo, que fundamenta la evolución fenoménica.

C2) Se demostró que la sintaxis del orden a priori de los numeros es trasladable a la sintaxis de la geometria, y que esta última, firmemente equipada con una sintaxis numérica natural, es trasladable a la sintaxis dé los hechos.

C3) El proceso abstracto de la evolución sintáctica culmina con la fundamentación del carácter logico de la sintaxis del lenguaje, a partir del cual podemos trazar el camino inverso bacia su punto de origen, mediante el análisis conceptual.

\section{Establecimiento de la significativa tautologia $\mathrm{H}^{2}$}

De acuerdo con los supuestos teoréticos de la mecánica cuántica (MC) y de la Teoría de la Relatividad (TR), se puede plantear fácilmente la siguiente ecuación, utilizando el modelo clásico Bohr-Sommerfeld del átomo $\mathrm{H}$, y la 
fórmula de Broglie que "reclama identidad sustancial y es igualadora de aspectos".

$$
\begin{aligned}
H^{2}= & 2 \pi e c V_{e} / V_{10} \text { (no dimensional) } \\
& (=\text { la significativa tautología “ } m T \text { ") }
\end{aligned}
$$

Leyenda:

$$
\begin{aligned}
e= & \text { carga eléctrica elemental } \\
= & 1,6021917 \times 10^{-20} \sqrt{\mathrm{g}} \sqrt{\mathrm{cm}} \\
& \left(=\sqrt{m_{e 0}} \sqrt{r_{e}}, \text { en donde } r_{c}\right. \text { es el radio del electrón) } \\
c= & \text { velocidad de la luz }=2,997925 \times 10^{10} \mathrm{~cm} / \mathrm{seg} \\
\nu_{c}= & \text { frecuencia de un fotón que en términos de energía } \\
& \text { corresponde a un electrón } \\
= & 1,23559 \times 10^{20} \mathrm{seg}^{-1} \\
V_{10}= & \text { la tensión eléctrical } 0^{10} \sqrt{\mathrm{g}} \mathrm{cm} \sqrt{\mathrm{cm}} / \mathrm{seg}^{2}(\text { voltios corregidos) }
\end{aligned}
$$

El que sea posible plantear la tautología $H^{2}$ demuestra que el clásico modelo mecánico-cuántico Bohr-Sommerfeld del átomo $\mathrm{H}$ no es -en lo que respecta a resultados numéricos- lógicamente inconsistente ni tiene tampoco el carácter de una aproximación inadmisible; dicho modelo tiene, más bien, el estatuto de un caso muy simple dentro de la mecánica cuántica no relativista.

\section{Una ilustradora transformación "interna" de la ecuación de onda}

En nuestro intento por demostrar que una descripción lógico-matemática correcta de la realidad (como la que presenta la MC) y una descripción conceptualmente correcta de la realidad deben necesariamente coincidir (deben ser las dos caras de una misma moneda), y no pueden diferir de tal suerte que la exactitud (claridad) de la primera implique la "irrazonabilidad" de la segunda, hemos ampliado la ecuación de Broglie:

$$
m c^{2}=b \nu
$$

con respecto a la energía del electrón, a:

$$
m_{e 0} c^{2}=b \nu_{e}=e V_{c}=\mu_{B} B_{\epsilon}
$$

esperando que, eventualmente, lleguemos a una "expresión unificada de la energía", que contenga (en uno solo) los cuatro aspectos fenoménicos posibles de la energía. 
Nuestra significativa tautología mT nos proporciona el medio para llegar a una expresión unificada de la energía, dado que ella nos permite ampliar el "significado (contenido) físico", sustituyendo el elemento matemático $2 \pi$.

$L a$ realidad fisica es, al parecer, tanto logaritmica natural como semejante a $\pi$.

A partir de:

$$
B_{e}=e H^{2} V_{10} / \mu_{B} \alpha
$$

(en donde $B_{c}$ es el campo magnético del electrón y $\mu_{B}$ el magnetón de Bohr) $\mathrm{y}$

$$
B_{\epsilon}=2 \pi e^{2} c \nu_{e} / \mu_{B} \alpha
$$

finalmente llegamos a:

$$
B_{c} \alpha / b v_{t} e c=8 \pi^{2} m_{c 0} / h^{2}
$$

sustituyendo $\mu_{B}$ por $e h / 4 \pi m_{c 0}$, y ampliando con el factor $1 / h$.

Y si ahora consideramos (la forma independiente del tiempo de la) ecuación de Schrödinger:

$$
\Delta \Psi+\left(8 \pi^{2} m_{e 0} / h^{2}\right)(E-U) \Psi=0
$$

resulta que el valor constante de la ecuación puede ser expresado como:

$$
8 \pi^{2} m_{c 0} / h^{2}=e H^{2} V_{10} \alpha / e h \nu_{c} c \mu_{B} \alpha=H^{2} V_{10} /\left(h \nu_{e} c\right) \mu_{B}
$$

sustituyendo $B_{\varepsilon}$ de acuerdo con (9). Y si remplazamos $H^{2} V_{10}$ de acuerdo con (4):

$$
H^{2} V_{10}=m_{e 0} c^{2} \alpha / e
$$

obtenemos:

$$
8 \pi^{2} m_{e 0} / h^{2}=m_{e 0} c^{2} \alpha / b \nu_{e} c e \mu_{B}=\alpha / e c \mu_{B}
$$

Si no ponemos en duda el hecho de que aquello que puede ser desmantelado debe existir, nuestra transformación del factor constante de la ecuación de onda, dado en (13), demuestra - por la incidencia de $H^{2} V_{10}$ - que el aspecto energético $e\left(H^{2} / \alpha\right) V_{10}$ (ver fórmula 4) es tan real como el aspecto $m_{c 0} c^{2}, \mathrm{y}$ que quizá desempeña un papel más importante que éste en la estructura de la cápsula atómica.

\section{Otro ejemplo del poder revelador de la "ontología concreta"}

El fenómeno de la superconductividad, es decir, el flujo de corriente eléctrica sin resistencia, que se da en ciertos metales y aleaciones a temperaturas cercanas al cero absoluto, va siempre acompañado de un flujo magnético. Como lo demostraron los físicos, este flujo magnético es cuantizado, es decir, que 
sus valores posibles están limitados por las leyes de la mecánica cuántica a un conjunto discreto de valores. Se encontró que la magnitud del quantum del flujo magnético se expresa convencionalmente por:

$$
b / 2 e=2,0678532 \times 10^{-7} \sqrt{\mathrm{gcm}} \sqrt{\mathrm{cm}} / \mathrm{seg}\left(\mathrm{Gauß} \mathrm{cm}^{2}\right)
$$

en donde " $b$ " es la cantidad de acción de Planck $\left(b=6,626196 \times 10^{-27}\right.$ $\mathrm{g} \mathrm{cm}^{2} / \mathrm{seg}$ ), y " $e$ " la carga eléctrica elemental (ver leyenda en la figura).

Se hizo del sorprendente factor 2 en el denominador el fundamento de una teoría de la superconductividad, basada en la idea de la acción conjunta de pares de electrones "congelados". Si bien la teoría ya ha sido corroborada, su carácter es meramente sintomático y de ahí que no pueda proporcionar a nuestro intelecto la posibilidad de una comprensión conceptualmente satisfactoria de ese fenómeno tan sorprendente. No tenemos la menor idea de cómo es que, por la acción de pares de electrones congelados, un flujo de electricidad que en temperaturas normales está inevitablemente atado a la aplicación artificial y mantenimiento de una tensión eléctrica, puede convertirse en un fenómeno autopreservado por tiempo ilimitado, tal como sucede en el caso del giro "eterno" de los electrones y otras partículas.

Nuestra comprensión del mundo como una materia sometida a las leyes a priori de la racionalidad nos ha sugerido que la tensión eléctrica existe como cualidad natural e inevitable dentro de la energía esencial (en reposo) del electrón en una magnitud definida. Si no nos aferramos al prejuicio de que la energía esencial del electrón es definitivamente irreductible, aunque se le puede desmantelar y hacer que se manifieste externamente a través de sus constituyentes en ciertas circunstancias artificiales, entonces no existe ninguna razón que nos impida pensar que los sucesos atemporales (eternos) del mundo microscópico pueden conducirnos a sucesos atemporales, mascroscópicamente observables, en ciertas circunstancias artificialmente provocadas.

Tal como quedó demostrado en el punto 7 de este trabajo, el considerar la energía esencial del electrón $m_{c o} c^{2}$ como un simple aspecto energético que, sorprendentemente, sólo aparece como fenómeno, en tanto que los otros tres aspectos son tan reales como el primero y pueden manifestarse conjuntamente como constituyentes estructurales de los fenómenos bajo ciertas circunstancias, la expresión convencional del quantum de flujo magnético (16), puede, de acuerdo con (4), ser transformado (sin que haya posibilidad de hacer una objeción lógica), de la siguiente manera:

$$
b / 2 e=H^{2} V_{10} / 2 \nu_{e} \alpha
$$

(en donde $2 \nu_{e}$ es la frecuencia de 2 fotones que, por su energía, corresponden a 2 electrones). 
Así pues, se demuestra que la tensión eléctrica $H^{2} V_{10}$ aparece como una parte esencial de la cantidad de flujo magnético, que se vuelve atemporal por la incidencia de la frecuencia " $2 \nu_{e}$ multiplicada por la constante estructural $\alpha$ ", como denominador.

Se puede también decir que " $h$, siendo la más pequeña porción eterna de todas las acciones posibles, puede despedir una cantidad atemporal natural de tensión eléctrica, bajo condiciones artificialmente provocadas, de magnitud $H^{2} V_{10}$, de acuerdo con:

$$
b \times 1 / 2 e=\left(2 \pi e^{2} c / \alpha\right) 2 e=\pi e c / \alpha=H^{2} V_{10} / 2 \nu_{\iota} \alpha .
$$

Si ahora consideramos que no puede haber corriente eléctrica sin la existencia de una tensión eléctrica, ni flujo magnético sin una corriente eléctrica, y viceversa, queda claro que el fenómeno de la superconductividad depende de la existencia y acción de la tensión eléctrica $H^{2} V_{10}$ que ocurre naturalmente y que se ha obtenido de la energía esencial de los electrones por el procedimiento artificial de hacer descender la temperatura a un punto cercano al cero.

Dado que se puede llamar a " $b$ " "lo último de la realidad" (Finkelnburg), pues es la única entidad física que no cambia en el curso del proceso lógicomatemático de la transformación Lorentz, y que, además, se le puede considerar como "energía que es eterna" debido a su dimensión "energía $\times$ tiempo", sólo “ $h$ ”, entonces, puede producir una tensión eléctrica que, al igual que ella, es atemporal, y que se manifiesta si se provocan las condiciones externas necesarias.

Creemos haber demostrado nuevamente que la correcta comprensión lógico-matemática de la realidad física, y su contraparte conceptual, siempre resultarán ser las dos caras de la misma moneda.

\section{Demostración de la racionalidad de la naturaleza mediante la posibilidad de} denotar fenoménicamente los fundamentos geométricos de la transformación de Lorentz

Desde un punto de vista filosófico, los fundamentos geométricos posibles de las fórmulas de transformación de la Teoría de la Relatividad Especial que hemos reconstruido nos dicen mucho más que sus "resúmenes" aritméticos.

La cantidad de energía eléctrica $(\mathrm{eV})$ que adquieren los electrones al ser acelerados por una tensión eléctrica, puede ser aritméticamente articulada de la siguiente manera:

$$
e V=m_{c 0} c^{2} / \sqrt{1-\left(v^{2} / c^{2}\right)}-m_{c 0} c^{2}
$$

(en donde "b" es la velocidad de los electrones acelerados). 
La energía cinética de un electrón es su energía eléctrica más la energía esencial (en reposo) de la partícula. Si tomamos el caso en que la magnitud de la energía cinética se vuelve exactamente el doble de la magnitud de la energía esencial, lo cual significa que " $e V=m_{e 0} c$ ", obtenemos una relación numérica muy simple:

$$
2=1 / \sqrt{1^{2}-0,75}
$$

Hemos llegado, así, a la velocidad “q" por la fórmula:

$$
\begin{aligned}
v & =c \sqrt{1-1 /\left(1+\left(e V / m_{e 0} c^{2}\right)\right)^{2}} \\
& =c \sqrt{1-1 /(1+1)^{2}} \\
& =c \sqrt{0,75}
\end{aligned}
$$

El factor $\sqrt{0,75}$ es equivalente al seno de un ángulo de $60^{\circ}$, entendiendo éste como el ángulo céntrico del círculo unitario; y esto significa que se puede interpretar el radio del círculo unitario como "velocidad de la luz" que - siendo la hipotenusa $\mathrm{H}$ del triángulo rectángulo inscrito en el círculo unitario- contribuye al establecimiento de la razón " $K_{c} / H$ ", la cual representa al seno del ángulo (en donde $K_{c}$ es el cateto opuesto al ángulo $\alpha$, que en nuestro caso es de $60^{\circ}$ ).

Podemos decir que el fenómeno relativista de la duplicación de la energía esencial es representado -o causado- por las relaciones geométricas resultantes de una descripción goniométrica (del seno) de un ángulo de $60^{\circ}$ (en el contexto del círculo unitario), de acuerdo con:

$$
\begin{aligned}
2\left(m_{e 0} c^{2}\right) & =1\left(m_{e 0} c^{2}\right) / \sqrt{H^{2}-K_{c}^{2}} \\
& =1\left(m_{e 0} c^{2}\right) / \sqrt{1-0,75} \\
& =1\left(m_{e 0} c^{2}\right) / 0,5
\end{aligned}
$$

Para lograr la aceleración de los electrones a una velocidad de $\sqrt{0,75} c$, que conduce a la duplicación de la energía esencial, es necesario aplicar una tensión eléctrica de $5,11041 \times 10^{4}$ voltios (corregidos), la cual, en nuestro enfoque ontológico, es igual a la tensión $\left(H^{2} / \alpha\right) \times V_{1}$ (en donde $V_{1}$ es la tensión eléctrica de $10^{1}$ ).

Lo que ahora resulta interesante es el hecho de que un fotón con una energía igual a $2 m_{e 0} c^{2}$, lanzado a un electrón en un ángulo de $60^{\circ}$, en el caso del efecto Compton, imparte, a su vez una velocidad de $\sqrt{0,75}$ al electrón, perdiendo $1 m_{e} c^{2}$ de energía y "regresando" $1 m_{c 0} c^{2}$ al electrón, con lo que se duplica la cantidad de energía esencial. Y esto implica que dos situaciones experimentales muy diferentes pueden ser representadas por exactamente el mismo contexto geométrico (ver figura 2). 


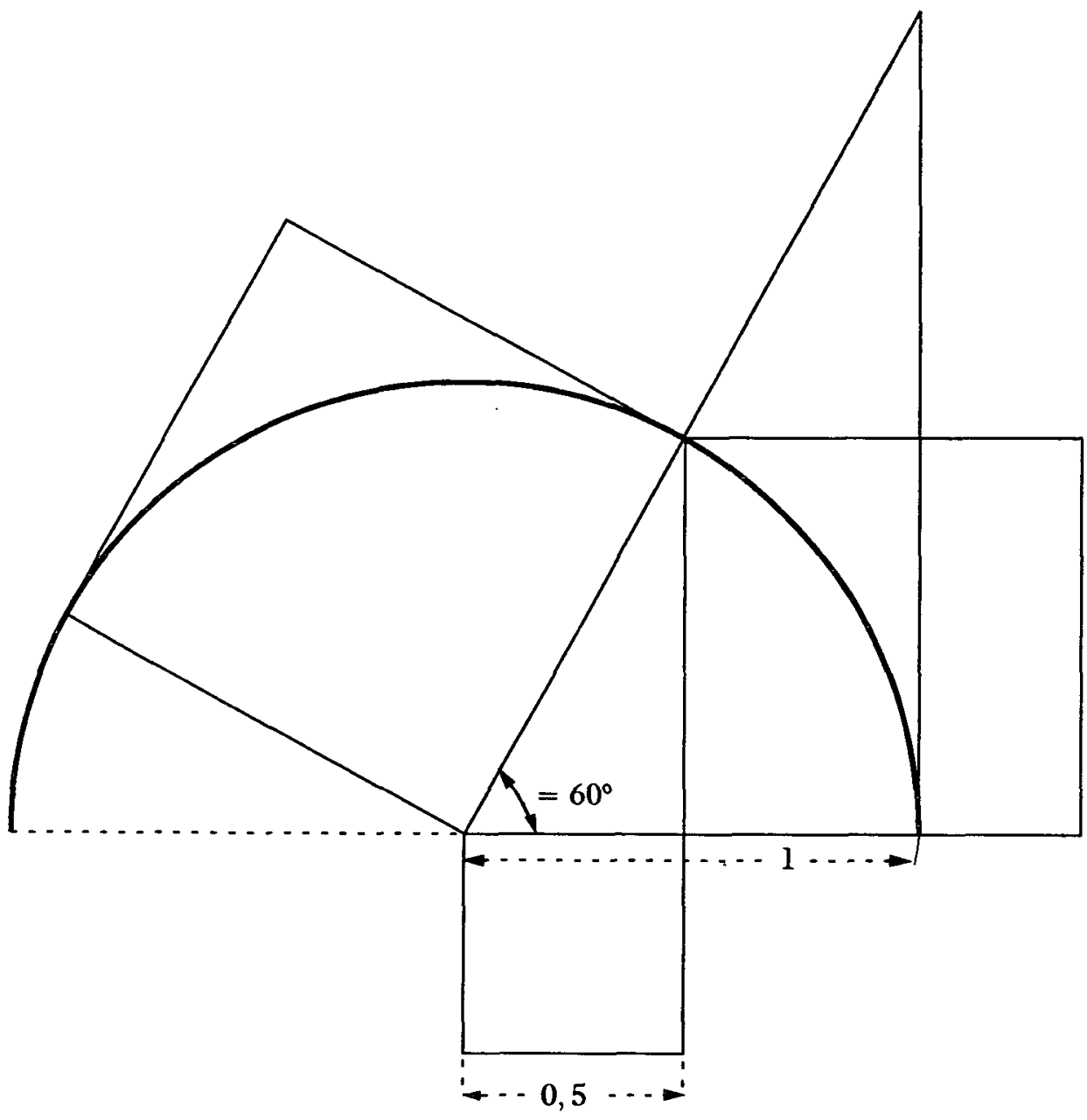

Figura 2

Ahora vamos a analizar hasta qué grado es completo el contexto geométrico "triángulo rectángulo con un ángulo de $60^{\circ}$ como ángulo céntrico del círculo unitario, incluyendo los cuadros formados por sus lados". La descripción trigonométrica de un ángulo que forma parte de un triángulo rectángulo no sería completa si no consideramos la función tangente junto con las funciones seno y coseno. En nuestro ejemplo, que se distingue por su simplicidad geometrica única, todos los números con los que hasta abora nos hemos encontrado, durante la descripción formal de los dos casos, son directamente representados 
como magnitudes y razones contextuales. (La "unidad de medida geométrica" del contexto es dada por el radio del círculo unitario cuya magnitud podemos elegir libremente.)

Dado que tenemos una denominación numérica completa de nuestro contexto, esto nos permite llegar a una denotación fenoménica completa del mismo, incluyendo las magnitudes espaciales de las dos longitudes de onda que intervienen, es decir, las longitudes de onda del fotón antes y después de su interacción con el electrón. (La unidad de las longitudes de onda es la longitud de onda Compton, $\lambda_{0}$, del electrón.)

Ahora bien, el cateto opuesto de nuestro ángulo dado con su descripción tangente ya está numéricamente denominado pero aún no ba sido fenoménicamente denotado. ¿Qué sucede si suponemos que la magnitud dada con este cateto opuesto debe tener un significado fenoménico, dado que la naturaleza debe utilizar la multiplicidad lógica del contexto, en su totalidad, para realizarse a sí misma? La callada implicación que se halla detrás de este "experimento mental" es la suposición de que el mundo está hecho más geométricamente y que, como tal, debe no sólo obedecer a las necesidades geométricas sino también hacer uso de la multiplicidad contextual de un contexto geométrico autocontenido, en su totalidad, y no puede - arbitrariamente- detenerse antes de ello.

En vista de la exactitud de esta suposición ontológica, queda claro que no hemos fabricado o inventado nuestro contexto (que, felizmente, se ajusta a la naturaleza), sino que lo hemos descubierto, como imagen logico-matemática inberente, en la naturaleza.

En el caso del cateto opuesto-tangente de nuestro ángulo lo que sucede es que su magnitud $(\sqrt{3})$ representa la razón: "longitudes de onda Compton del electrón/longitud de onda de su onda matérica, a una velocidad de $\sqrt{0,75} c$.

Se puede decir que: se habría podido predecir la existencia de algo como las "ondas materiales" a condición de que el mundo fuera un caso lógicomatemático, es decir, que tuviera un aspecto abstracto en el cual se inscribieran las imágenes lógicas de una multiplicidad determinada, las cuales tendrían que ser realizadas in toto fenoménicamente. Podemos asimismo sostener que nuestra suposición ontológica nos permite dibujar la frontera entre la multiplicidad concebible a priori y la logicamente posible, de la realidad física.

Nota: El haber tomado en consideración el aspecto de "angularidad esencial" de las interacciones físicas demuestra que la transformación Lorentz es una condición necesaria de la estructura dinámica del mundo, mientras que la llamada transformación de Galileo no es sino una fantasía imposible.

\section{Resumen cientifico preliminar}

La física cuántica está — como acertadamente dijo uno de nuestros críticos 
(el moderador) - completa, lo cual significa que "ha llegado a su fin", en la medida en que, por razones lógico-matemáticas, ya no es posible seguirla desarrollando consistentemente (afirmación de Kochen, Specker y Giuntini).

La pregunta planteada por Stephen Hawking: "jacaso se anuncia el fin de la física teórica?" resulta, pues, algo más que simple retórica.

A la mecánica ondulatoria (Schrödinger) y a la mecánica cuántica (Heisenberg) hoy en día se les llama, por ser lógicamente equivalentes, mecánica cuántica (MC). Se puede describir a la MC como aquella parte de la teoría cuántica (TC) en la cual sólo las velocidades muy por debajo de la velocidad de la luz son significativas, y en la cual el número de partículas no varía en función del tiempo. En la teoría cuántica de los campos (TCC), por el contrario, se presupone la posibilidad de la generación y aniquilación de partículas. Los resultados y predicciones de la $\mathrm{MC}$, incluyendo su ampliación relativista (Dirac), merecen - como afirma Finkelnburg, el viejo maestro de la microfísicaabsoluta confianza. Se tiene menos confianza en la TCC que en la MC dado que aquélla, finalmente, llega a afirmar la posibilidad de la "existencia virtual", es decir, "existencia en efecto aunque no en un hecho real". Dado que la TCC debe sostener que existe una ondulación en el número de partículas existentes, en función del tiempo, ésta entra en conflicto con ciertos resultados de la MC que, de manera obvia, concuerdan totalmente con los casos y sucesos de la realidad física. En este sentido, la TCC no parece ofrecer una salida a los problemas y limitaciones de la MC.

La estructura de la MC, si se considera el grado hasta el cual es completa conceptualmente, no parece satisfactoria, debido a que, por ejemplo, el fenómeno físico fundamental de la "tensión eléctrica" no aparece explícitamente en la ecuación de onda (no relativista) fundamental.

La transformación del factor constante de la ecuación de onda, que fue posible hacer mediante nuestro procedimiento filosófico (13), demuestra que el fenómeno de la tensión eléctrica siempre ha formado parte de los fundamentos de la mecánica cuántica como una de sus invariantes básicas. Lo mismo sucede con la velocidad de la luz que, si bien no había desempeñado un papel "externo" en la MC no relativista, aparece como elemento constitutivo (13, 15), invariable, de dicha teoría.

Finalmente, nuestras consideraciones conceptuales demostraron (15) que $\alpha$, en tanto factor trascendente y determinante de lugar, "no ha perdido su importancia" en el curso de la evolución de la MC, sino que sigue siendo una invariante inherente a la teoría.

Podemos decir que, si bien al parecer es imposible que la MC amplíe externamente su estructura teórica, mediante la extensión del aparato matemático, ha probado ser internamente extensiva. La extensibilidad interna de la MC, cuya existencia hemos demostrado, descansa en el hecho de que el elemento matemático $2 \pi$, en sí mismo, representa una parte bien determinada de la 
realidad física, el cual se manifiesta al efectuar un desmantelamiento. Aí pues, queda demostrado que la comprensión de la realidad fisica puede avanzar y ser profundizada utilizando "la forma en que las humanidades resuelven los problemas", para alcanzar un conocimiento sobre la estructura del mundo que sobrepase, con mucho, todas las posibilidades del razonamiento científico (convencional).

La tarea cultural de la ciencia, a saber, la demostración de que la realidad física depende de un a priori metafísico, no podía ser realizada por los científicos realistas - a los cuales la simple idea de la existencia de la sintaxis lógica del lenguaje les parece una fantasía extraña y exótica- sino sólo a través de un proceso de razonamiento filosófico tanto libre de prejuicios como comprensivo.

Es ahora cuando toma forma la posibilidad de elegir cuál es el tipo de Weltanschauung que sostiene la verdadera naturaleza de la naturaleza.

\section{Resumen filosofico}

Hemos logrado hacer visible la constante universal $\alpha$ utilizando "la forma de razonar de las humanidades", es decir, hemos logrado abordar a $\alpha$ desde el lado del aspecto metafisico del mundo, por la mera operación del pensamiento, sin dejar de considerar, no obstante, los hechos físicos en concreto. $\alpha$ aparece como el vinculo unico entre los aspectos físico y metafísico del mundo. ( $\alpha$ tiene tanto un polo metafísico como un polo físico. Con respecto al lenguaje objeto, el origen y relación de los dos polos son recíprocos.)

Afirmamos que hemos logrado demostrar:

i) la existencia de un orden numérico a priori del mundo, que es idéntico al sistema de los logaritmos naturales;

ii) la existencia de una "lógica de los hechos";

iii) que la lógica de los hechos depende del orden a priori de los números; y, simultáneamente, hemos demostrado:

0) la existencia de un método ontológico, que

00) trasciende el "razonamiento científico" convencional, y que lleva a 000) constatar una evolución sintáctica abstracta de la realidad fenoménica.

Dada la inexpresabilidad de la oración: "Se puede hablar de una concordancia justificable (Gemäßheit) entre las magnitudes de las medidas para diferentes entidades - como las magnitudes de las unidades para masa, longitud y tiempo-", resulta lógicamente imposible que exista un sistema ideal de medición - que sea preferido por la naturaleza. Todos los sistemas de medición "semejantes a uno" no sólo tienen el mismo valor para una correcta descripción de la realidad física, sino que son logicamente equivalentes. 
El plural del concepto "sistema de medición" no existe. Y ésta es la razón por la que es imposible traducir un sistema de medición que ya es "semejante a uno" (eins-mäßig) a cualquier otro, haciendo del primero el lógicamente ideal, por la "semejanza a uno" (Eins-mäßigkeit), en tanto que el otro pasa al último lugar por la "semejanza a uno-punto-x". La única condición lógica para que un sistema de medición pueda ser utilizado exitosamente en la demostración de que el mundo fenoménico depende de su aspecto metafísico, es la "semejanza a uno". Expresado inversamente: todo sistema de medición que no se base en la más pequeña unidad "uno" es irracional, dado que no permite expresar la necesidad lógica de la concordancia con la "semejanza a uno" del orden a priori dado de los números naturales. La sorpresa es que:

Los sistemas de medición semejantes a uno, libremente seleccionados, nunca son arbitrarios.

Traducción de LoRena MuRILlo S.

REVISIÓN DE SERGIO MARTÍNEZ 